JOURNAL OF MODERN OPTICS, 2000, vol. 47, NO. 8, 1339-1345

\title{
Influence of the gaze-stabilizing eye movements on the quality of the retinal image of the human eye
}

\author{
A. M. PONS†, A. LORENTE $\dagger$, C. ILLUECA \\ J. M. ARTIGAS $\dagger$ and A. FELIPE $\dagger$ \\ $\dagger$ Dept Interuniversitari d'Òptica, Universitat de València, \\ Dr Moliner 50, 46100 Burjassot, Valencia, Spain \\ $\$$ Dept Interuniversitari d'Optica, Universitat d'Alacant, \\ Apdo. 9903080 San Vicent del Raspeig, Alicante, Spain
}

(Received 20 September 1999; revision received 12 January 2000)

\begin{abstract}
In this work we have studied the influence of the gaze stabilizing movements of the eye on the quality of the retinal image of the human eye obtained by double pass methods. The results obtained agree with the expected differences between the coherent and incoherent behaviour of the optical system of the eye. The movements-free retinal image is obtained from a typical retinal image by considering a filter function in the frequency domain which characterizes the effect of the considered movements.
\end{abstract}

\section{Introduction}

The gaze-stabilizing task is carried out by the visual system through the action of different types of eye movements. In general, it is accepted that two main types of eye movement are observed during maintained fixation: tremors and small drift saccades [1]. Tremors are high frequency movements $(70-80 \mathrm{~Hz})$ with mean peakto-peak amplitude of 30 arcsec $[2,3]$. Due to the low amplitude of these movements, their influence on vision is irrelevant, as was confirmed by Packer and Williams [4]. The small-drift saccades ('microsaccades') are low frequency movements $(2-5 \mathrm{~Hz})$ with a peak-to-peak amplitude lower than 5 arcmin $[5,6]$ and they are supposed to be involved in the prevention of the image fading [1]. Different studies [7-9] have been developed to determine the aim of the existence of these movements, but up until now, no useful role for small saccades has been justified.

The determination of the optical quality of the human eye has been generalized by the use of the double pass method [10-12]. In this method, a biteboard is used to control the head movements during the record of the retinal image. It is known that small saccades are prevalent in target fixation when an artificial head support is used $[13,14]$ and the use of cyclopegic or midriatic drugs do not minimize the presence of these movements [15].

If temporal integration techniques are used in the retinal image recording process $[11,12,16]$, the importance of these movements becomes evident: they are responsible for the loss of coherence in the second pass of the process, blurring the individual coherent images [17], thus losing the speckle. In fact, these movements 
act as a low-pass filter over the retinal image in the same way as mechanical vibrations [18] and can be eliminated following similar procedures to the ones used in the restoration of motion degraded images $[19,20]$.

The aim of this work is to obtain a movements-free retinal image, obtaining a filter function in the frequency domain to characterize the eye movements during the recording interval.

\section{Methods}

The retinal images recorded by the double pass technique are obtained, generally, by the use of a temporal integration interval of $T$ seconds. During this interval, if the refresh rate of the CCD (charge-coupled device) is $t_{0}$, a total of $N=T / t_{0}$ images are integrated. In order to simulate the effect of eye movements, we can suppose that, during the $T$ interval, a total of $M_{\mathrm{T}}$ tremors and $M_{\mathrm{S}}$ small saccades occur. Knowing the mean frequencies of these movements, $f_{\mathrm{T}}$ and $f_{\mathrm{S}}$ :

$$
M_{\mathrm{T}}=T f_{\mathrm{T}} \quad \text { and } \quad M_{\mathrm{S}}=T f_{\mathrm{S}}
$$

Each movement has a random amplitude within a normal distribution of means $\left\langle D_{\mathrm{T}}\right\rangle$ and $\left\langle D_{\mathrm{S}}\right\rangle$, with random directions $\theta_{\mathrm{T}}$ and $\theta_{\mathrm{S}}$.

In order to obtain how a point image is deformed by these movements, we can simulate the final result by applying random spatial displacements around the fixation point with the same amplitude and frequency as the tremors and saccades.

If $I(x, y)$ is an ideal point object, defined by a Dirac delta function

$$
(x, y)=\delta(0,0),
$$

after one general eye movement, the image has been 'moved' to a new position defined by their polar coordinates $(D, \theta)$ :

$$
I^{\prime}(x, y)=\delta(D \cos \theta, D \sin \theta) .
$$

During the displacement between the zero position (fixation point) and the new position, a total of $1 / t_{0} f_{\mathrm{i}}$ images are integrated by the $\operatorname{CCD}\left(f_{\mathrm{i}}\right.$ being the temporal frequency of the spatial movement considered). Following the Listing law [21], we can suppose a linear movement between these two points, so the resulting image will be:

$$
\sum_{k=1}^{1 / t_{0} f_{\mathrm{i}}} \delta(d k \cos \theta, d k \sin \theta)
$$

$d$ being

$$
d=D_{\mathrm{i}} t_{0} f_{\mathrm{i}} .
$$

After the $T$ interval, the total image will be:

$$
\begin{aligned}
M(x, y)= & \sum_{i=1}^{M_{\mathrm{T}}} \sum_{k=1}^{1 / t_{0} f_{\mathrm{i}}} \delta\left(d_{i} k \cos \theta, d_{i} k \sin \theta_{i}\right) \\
& +\sum_{j=1}^{M_{\mathrm{S}}} \sum_{k=1}^{1 / t_{0} f_{\mathrm{i}}} \delta\left(d_{j} k \cos \theta_{j}, d k \sin \theta_{j}\right),
\end{aligned}
$$


where

$$
d_{i}=D_{\mathrm{i}}^{\mathrm{S}} t_{0} f_{\mathrm{s}} \text { and } d_{j}=D_{\mathrm{i}}^{\mathrm{T}} t_{0} f_{\mathrm{T}} .
$$

This image is the result of applying the eye movements over a $\delta$ signal. If we want to characterize the spatial eye movements as a filter function in the spatial frequencies' domain, we must take the Fourier transform of $M$ :

$$
f_{\mathrm{m}}(u, v)=\mathfrak{J}\{M(x, y)\} .
$$

Due to the random nature of eye movements, if we want to obtain a general filter function, we must average over a large number of $f_{\mathrm{m}}$ functions, deriving the filter function as the mean of this individual random $f_{\mathrm{m}}$ function, $\left\langle f_{\mathrm{m}}\right\rangle$.

Once obtained the filter function $\left\langle f_{\mathrm{m}}\right\rangle$, we can simulate a movements-free image filtering in the spatial frequencies' domain the spatial spectrum of an actual image with this function. If $I(x, y)$ is an actual retinal image, obtained after a $T$ interval of temporal integration and its spectrum is:

$$
\tilde{I}(x, y)=\mathfrak{J}\{I(x, y)\},
$$

then the spatial spectrum of the free-moments image will be:

$$
\tilde{I}^{*}(x, y)=\frac{\mathfrak{I}\{I(x, y)\}}{\left|\left\langle f_{\mathrm{m}}\right\rangle\right|} .
$$

\section{Results}

Computer simulations for $f_{\mathrm{m}}$ were obtained for a typical integration time of $750 \mathrm{~ms}$ and mean frequencies of $75 \mathrm{~Hz}$ for tremors and $3.5 \mathrm{~Hz}$ for small saccades. The mean amplitude considered was 5 arcmin for small saccades and 30 arcsec for tremors. A total of 1000 individual $f_{\mathrm{m}}$ functions were averaged to obtain the final $\left\langle f_{\mathrm{m}}\right\rangle$ filter function, showed in figure 1 . As can be seen, the $\left\langle f_{\mathrm{m}}\right\rangle$ function is a lowpass filter in the spatial frequencies' domain so a blurring of the original image must be expected after applying the filter.

In the double pass method, the registered images are the result of two cascade filters: the eye optics modulation transfer functions (MTF) and the eye movements' filter. If we apply equation (10) to the spatial frequency spectrum of an actual image, a free-movements image will be obtained. Figure $2(a)$ represents an actual image obtained by the double pass method, following the experimental protocol and set-up of Lorente et al. [11]. Figure $2(b)$ represents the result of applying the inverse Fourier transform over the result of equation (10). As can be seen, the resulting image recovers the speckle present in the first coherent pass of the double pass method [17].

From this result, it is evident that we can obtain both the coherent and the incoherent MTF of the eye, using the procedure proposed by Lorente et al. [17]. If $I(x, y)$ is the incoherent retinal image, the incoherent MTF of the human eye will be:

$$
H(u, v)=(|\mathfrak{J}\{I(x, y)\}|)^{1 / 2}
$$

and, in the case of a coherent image (in our case, a free-movements image), $I^{*}(x, y)$, 


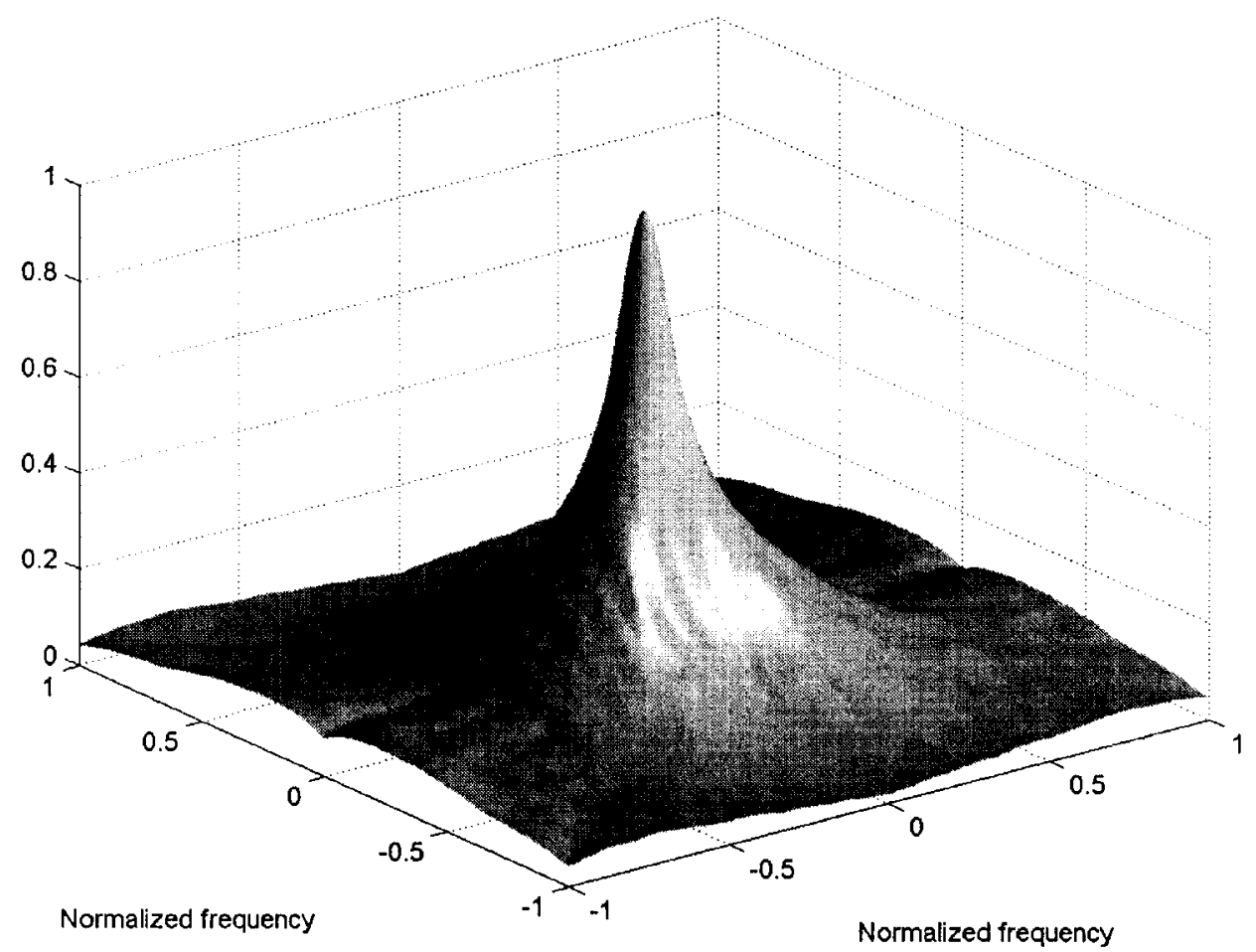

Figure 1. Final filter function obtained after averaging over a large number of individual $f_{\mathrm{m}}$ functions.

the coherent MTF of the human eye, can be derived as:

$$
H^{*}(u, v)=\left|\mathfrak{J}\left\{[I(x, y)]^{1 / 2}\right\}\right| .
$$

If we apply equations (11) and (12) to the results of figures $2(a)$ and $(b)$, we must obtain the incoherent and coherent MTFs respectively. The result of these operations can be seen in figure 3 . As follows from the theory of coherent imaging [22], the coherent MTF has a lower cut-off frequency than the one resulting from the incoherent MTF. It can also be shown how the area below the incoherent MTF is greater than the area of the coherent MTF.

\section{Conclusions}

The proposed methodology allows one to obtain experimental results of coherent imaging by the human eye, avoiding the use of an anaesthetic drug which have a midriasys and accommodation paralysis as secondary effects [15]. The use of only cyclopegic or midriatic drugs do not avoid the presence of eye movements so, when these drugs are used, an incoherent treatment of the resulting image must be carried out.

This coherent treatment of the optics of the eye can be applied to validate the studies of the performance of the visual system under coherent illumination conditions [23]. 


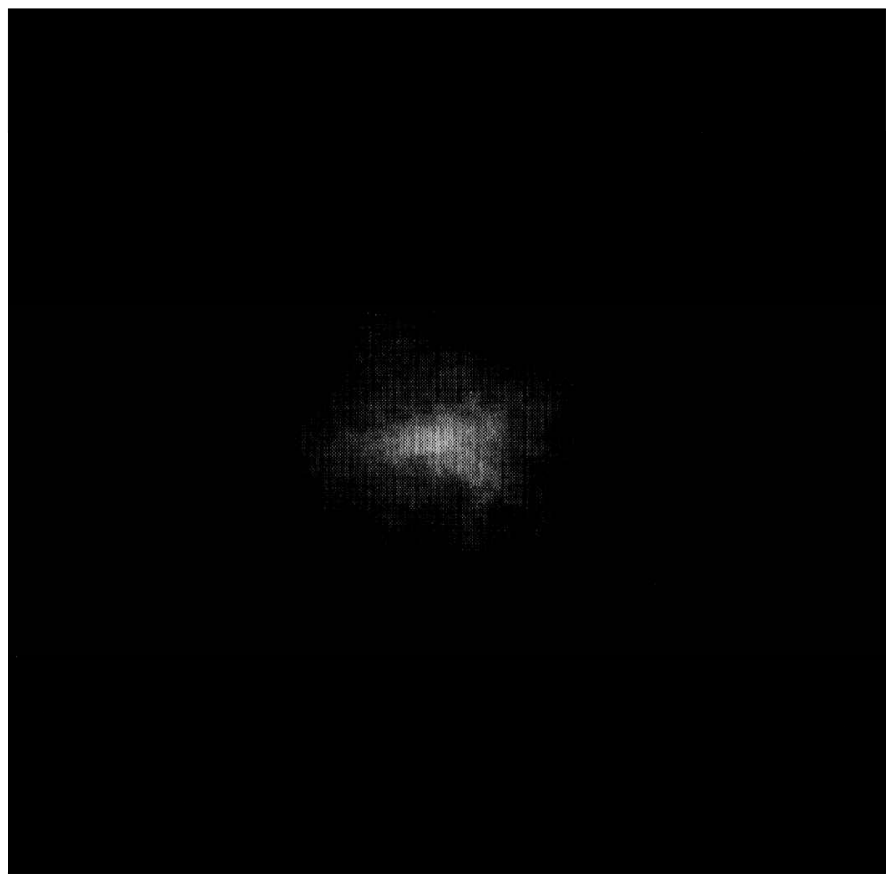

(a)

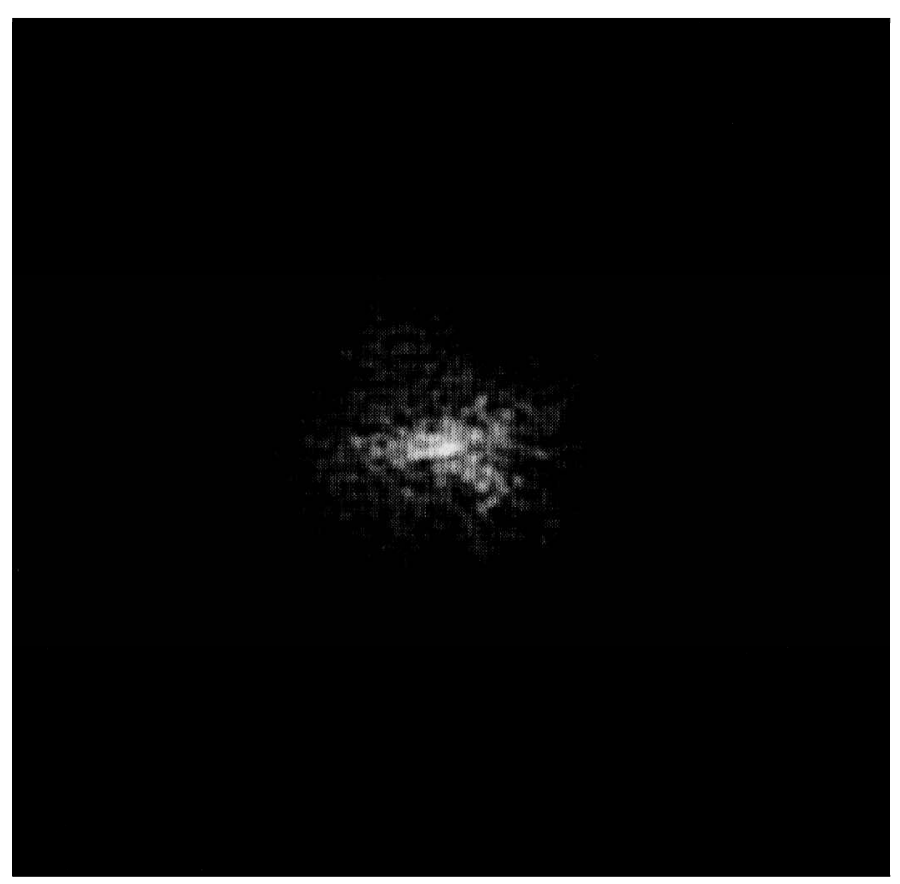

(b)

Figure 2. (a) Retinal image obtained from a double pass measurement. (b) Freemovements retinal image calculated after applying the filter function $\left\langle f_{\mathrm{m}}\right\rangle$. 


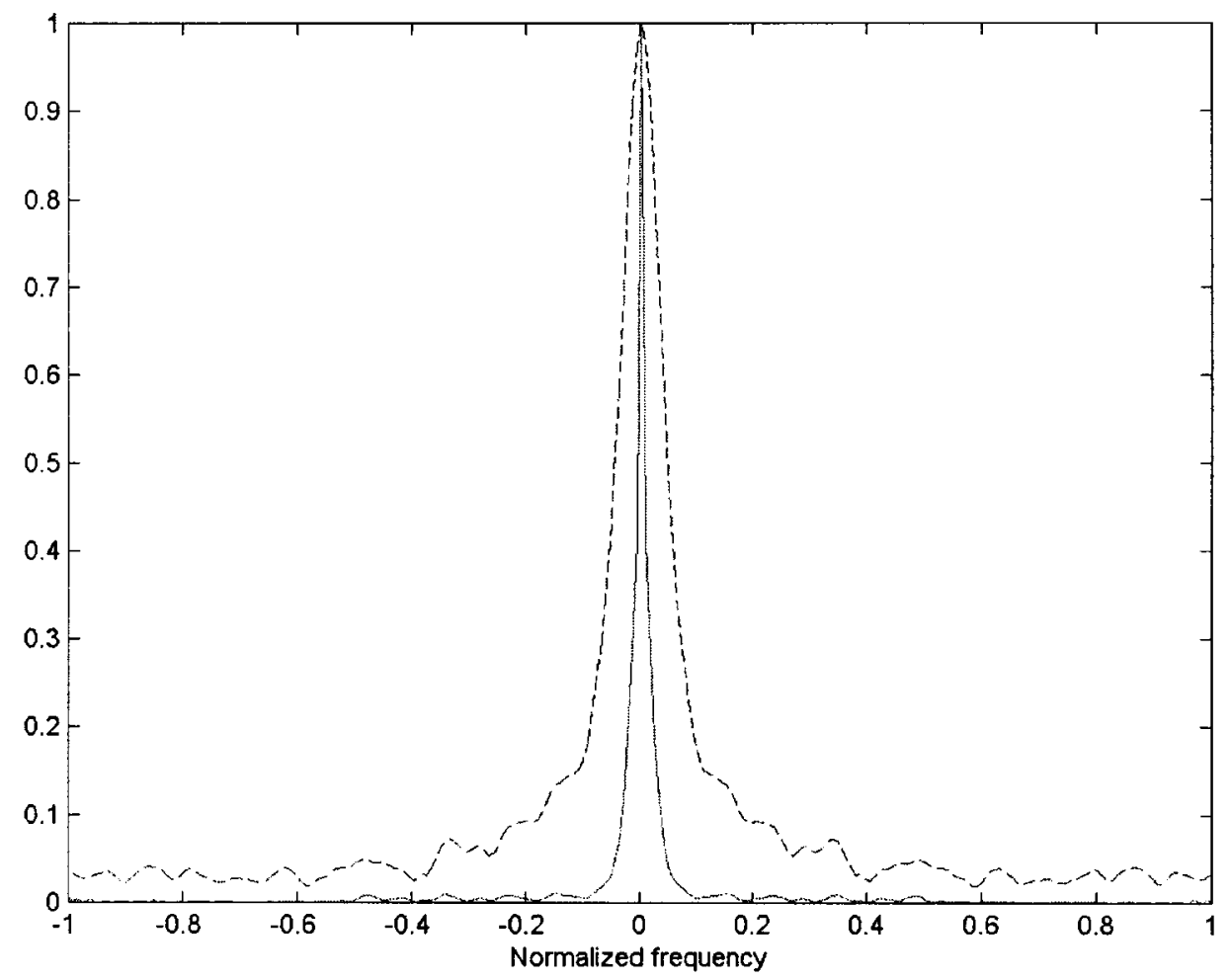

Figure 3. Modulation transfer functions derived from figures $2(a)$ and $(b)$ : the MTF for the retinal image are shown as continuous line. The dashed line corresponds to the image quality of the free-movements MTF.

\section{References}

[1] Kowler, E., 1991, Eye Movements, edited by R. H. Carpenter (London: MacMillan Press).

[2] Ratliff, F., and Riggs, L. A., 1950, J. Exp. Psychol., 40, 687.

[3] Ditchburn, R. W., and Ginsborg, B. L., 1953, J. Physiol., 145, 98.

[4] Packer, O., and Williams, D. R., 1990, Invest. Ophthalmol. Vision Sci., Suppl. 31, 494.

[5] Cornsweet, T. N., 1956, J. opt. Soc. Am., 46, 987.

[6] Krauskopf, J., Cornsweet, T. N., and Riggs, L. A., 1960, J. opt. Soc. Am., 50, 572.

[7] Ditchburn, R. W., 1980, Vision Res., 20, 271.

[8] Kowler, E., and Steinbman, R. M., 1980, Vision Res., 20, 273.

[9] Kowler, E., Pizlo, P., Zhu, G. L., Erkelens, C. J., Steinman, R. M., and Collewijn, H., 1990, The Head-Neck Sensory Motor System: Evolution, Development, Disorders and Neuronal Mechanisms, edited by A. Berthoz, W. Graf and P. P. Vidal (New York: Oxford University Press).

[10] Santamaria, J., Artal, P., and Bescós J., 1987, J. opt. Soc. Am. A, 4, 1109.

[11] Lorente, A., Pons, A. M., Malo, J., and Artigas, J. M., 1997, Ophthalmic physiol. Optics, 17, 267.

[12] Pons, A. M., Lorente, A., Albarrán, C., Montés, R., and Artigas, J. M., 1998, Ophthalmic physiol. Optics., 18, 40.

[13] Steiman, R. M., and Collewijn, H., 1980, Vision Res., 20, 415.

[14] Skavensky, A. A., Hansen, R. H., Steinman, R. M., and Weinterson, B. J., 1979, Vision Res., 19, 675. 
[15] Jahnus, S. D., Pagano, V. T., Bartlett, J. D., 1989, Clinical Ocular Pharmacology, edited by J. D. Bartlett and S. D. Jaanus (Boston: Butterworths), pp. 69-148.

[16] Artal, P., Marcos, S., Navarro, R., and Williams, D. R., 1995, J. opt. Soc. Am. A, 12, 1951.

[17] Lorente, A., Artigas, J. M., Felipe, A., Pons, A. M., and Gomez, J., 1999, J. mod. Optics, 46, 1079.

[18] Wulich, D., and Kopeika, N. S., 1987, Opt. Eng., 26, 529.

[19] Barnard, K. J., White, C. E., and Absi, A. E., 1999, Appl. Optics, 38, 1942.

[20] Stern, A., and Kopelka., N. S., 1999, Appl. Optics, 38, 757.

[21] Alpern, M., 1969, The Eye, Vol. 3, edited by H. Davson (New York: Academic Press).

[22] Goodman, J. W., 1968, Introduction to Fourier Optics (New York: McGraw Hill).

[23] Buades, M. J., Felipe, A., Artigas, J. M., Pons, A. M., 1997, J. opt. Soc. Am. A, 14, 972. 